\title{
Étude numérique du comportement en fatigue à grand nombre de cycles d'agrégats polycristallins de cuivre
}

\author{
Camille Robert $^{1,2, a}$, Nicolas Saintier ${ }^{2}$, Thierry Palin-Luc ${ }^{2}$ et Franck Morel ${ }^{1}$ \\ 1 Arts et Métiers ParisTech, LAMPA, 2 bd du Ronceray, 49035 Angers Cedex, France \\ 2 Arts et Métiers ParisTech, I2M - UMR CNRS 5295, Université Bordeaux 1, esplanade des Arts et Métiers, \\ 33405 Talence Cedex, France
}

Reçu le 3 mai 2011, accepté le 11 mai 2011

\begin{abstract}
Résumé - Une analyse du comportement en fatigue multiaxiale à grand nombre de cycles est conduite à travers la simulation numérique d'agrégats polycristallins. Le matériau métallique choisi est un cuivre de microstructure CFC. Le VER, constitué d'un ensemble de 300 grains d'orientation aléatoire équiprobable et de géométrie équiaxe, est sollicité au niveau de la limite de fatigue définie pour un nombre de cycles de $10^{7}$ cycles, le but étant de calculer les quantités mécaniques relatives à l'échelle mésoscopique (moyenne à l'échelle du grain) après stabilisation du comportement cyclique local. Les résultats de la simulation montrent la grande dispersion des variables mécaniques à cette échelle mésoscopique. Une analyse statistique de la réponse de l'agrégat est proposée pour différents chargements purement alternés : traction, torsion, traction-torsion combinées en-phase. Grâce à la connaissance des quantités mécaniques locales pour un nombre suffisamment grand de microstructures différentes, une analyse critique de certains critères d'endurance multiaxiaux (Crossland et Dang Van) est conduite.
\end{abstract}

Mots clés : Fatigue / simulation numérique / agrégats polycristallins

\begin{abstract}
Numerical study of high cycle fatigue behaviour of copper polycrystalline aggregates. An analysis of high cycle fatigue behaviour is undertaken via the numerical simulation of polycrystalline aggregates. The metallic material chosen for investigation is Copper, which has a FCC crystalline structure. The REV, which is composed of 300 randomly orientated equiaxed grains, is loaded at the fatigue limit determined at $10^{7}$ cycles. The aim is to calculate the mechanical quantities at the mesoscopic scale (average quantities in the grains) after cyclic stabilisation has been achieved. The results highlight the fact that the mechanical quantities at this scale have a large scatter. A statistical analysis of the response of the aggregate for different loading conditions (tensile, torsion, and in-phase tension-torsion) is done. Thanks to the sufficiently large number of different microstructures investigated, a critical analysis of the Dang Van and Crossland multiaxial fatigue criteria has been undertaken, using the local mechanical quantities.
\end{abstract}

Key words: Fatigue / numerical simulation / polycrystalline aggregates

\section{Introduction}

Le recours à la simulation numérique d'agrégats polycristallins a permis, depuis les vingt dernières années [1], d'apporter un éclairage nouveau sur le lien entre les comportements mécaniques à l'échelle des grains et la réponse globale à l'échelle du VER. Même si les comportements mécaniques sous chargement monotone ont principalement été ceux abordés, des études dédiées au comportement en fatigue sont de plus en plus fréquentes [2].

\footnotetext{
a Auteur pour correspondance :

camille.robert@ensam.eu
}

Quel que soit le type de comportement étudié, la question de la représentativité de l'agrégat numérique utilisé pour la simulation se pose néanmoins toujours. Parallèlement au développement de ces approches, la prise en compte du rôle des hétérogénéités microstructurales dans les modélisations en fatigue, et en particulier les critères d'endurance multiaxiaux, est maintenant possible à l'aide de certains travaux récents $[3,4]$. Les modèles proposés s'attachent à rendre compte le plus simplement possible de l'effet de la variabilité de comportement mécanique à l'échelle de la microstructure. L'hypothèse du maillon le plus faible est souvent utilisée quand il 


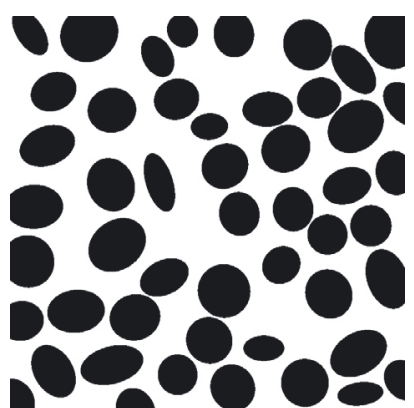

Ellipses

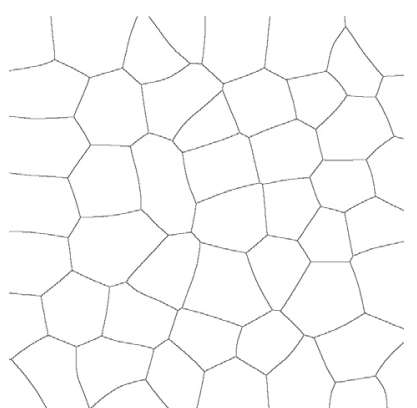

Watershed

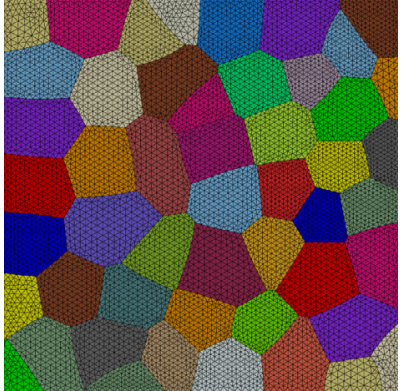

Maillage

Fig. 1. Principales étapes utilisées pour générer le maillage de l'agrégat polycristallin.

s'agit de traiter du domaine de la fatigue à grand nombre de cycles. Cette étude s'inscrit donc dans le cadre de la meilleure compréhension du rôle des hétérogénéités microstructurales sur la réponse en fatigue multiaxiale à grand nombre de cycles et ceci à travers la simulation numérique d'agrégats polycristallins. Les principaux volets abordés sont :

- le rôle de la morphologie et de l'orientation des grains sur la réponse mécanique sous chargement cyclique pour des niveaux proches de la limite de fatigue à $10^{7}$ cycles ;

- l'analyse de la distribution de certains paramètres mécaniques mésoscopiques (cission résolue, contrainte hydrostatique, contrainte normale) induits pour différents agrégats numériques et par différents chargements cycliques macroscopiques purement alternés (traction, torsion, traction-torsion combinées en phase) ;

- la capacité de certains critères d'endurance multiaxiaux (Crossland, Dang Van) à rendre compte des tendances observées au travers des résultats de la simulation numérique.

\section{Topologie des agrégats}

La génération de maillage de microstructures polycristallines a fait l'objet de nombreuses études. Parmi les méthodes les plus utilisées, la plus simple consiste à créer des grains de topologie identique puis à les dupliquer de sorte à remplir tout le volume de la microstructure. Cette méthode relativement simple permet d'obtenir rapidement des microstructures numériques ainsi que des résultats qualitatifs. Cette méthode est utilisée par exemple dans [2] avec une topologie de grains hexagonale. De nombreux auteurs [5-7] ont recours aux polyèdres de Voronoï pour obtenir des microstructures plus proches de la réalité. Ce type de topologie permet notamment d'avoir une distribution de la taille des grains. Il est également possible d'utiliser plusieurs polyèdres de Voronoï pour un grain [8], ce qui permet une géométrie plus proche de la réalité. Enfin des méthodes plus élaborées peuvent être utilisées comme dans [9] ou [10] pour avoir des frontières courbes et ainsi une microstructure encore plus représentative.
Une microstructure 2D sera utilisée dans ce travail. Afin de pouvoir paramétrer au mieux la géométrie des grains, l'idée utilisée dans [9] est reprise. Elle consiste, à partir de distributions particulières de taille, allongement et orientation d'ellipses, à appliquer, grâce à l'algorithme « watershed », une dilatation de ces mêmes ellipses jusqu'à obtenir une géométrie particulière de la microstructure. Enfin, pour intégrer cette morphologie dans un modèle $\mathrm{CAO}$, la géométrie de la frontière des grains, entre deux points triples, est approximée par une courbe de Bézier. Le modèle CAO est alors discrétisé par des éléments triangulaires. Un exemple des différentes étapes appliquées est présenté figure 1.

\section{Comportement du matériau}

La loi de comportement cristalline utilisée dans ce travail a été introduite par Meric et Cailletaud [1]. Elle est couramment utilisée pour la simulation numérique d'agrégats polycristallins [11]. Les relations constitutives sont définies par les équations (1)-(6). Le modèle est utilisé dans le cadre des petites perturbations, avec un comportement élastique orthotrope (paramètres $C_{11}$, $C_{22}$ et $\left.C_{12}\right) \cdot \gamma^{\mathrm{s}}$ représente le glissement plastique sur le syst- ème $\mathrm{s}$, et $\nu^{\mathrm{s}}$ le glissement plastique cumulé sur le système s. L'écrouissage isotrope est défini sur chaque système de glissement par la variable $r^{\mathrm{s}}$, et l'écrouissage cinématique par la variable $x^{\mathrm{s}}$. $c$ et $d$ sont des paramètres de la loi de comportement. Le tenseur d'orientation $\boldsymbol{m}^{\mathrm{s}}$, qui est calculé sur chaque système de glissement $s$ par le produit tensoriel de la normale au plan de glissement $\boldsymbol{n}^{s}$ et la direction de glissement $\boldsymbol{l}^{\mathrm{s}}$, est utilisé pour le calcul de la cission résolue $\tau^{\mathrm{S}}$ et le tenseur du taux de déformation plastique, $\dot{\varepsilon}_{\mathrm{p}}$. Les deux paramètres matériau, $K$ et $n$, représentent la sensibilité à la vitesse de déformation. Enfin, les paramètres $r_{0}, Q$ et $b$ (ainsi que les composantes $h_{\mathrm{rs}}$ de la matrice d'interaction) sont utilisés pour la modélisation de l'écrouissage isotrope.

Les paramètres matériau utilisés, identifiés sur un cuivre à haute pureté par Gérard et al. [12], sont présentés dans la tableau 1.

$$
\dot{\gamma}^{\mathrm{s}}=\left(\frac{\left\|\tau^{\mathrm{s}}-x^{\mathrm{s}}\right\|-r^{\mathrm{s}}}{K}\right)^{n} \operatorname{sign}\left(\tau^{\mathrm{s}}\right)=\dot{\nu}^{\mathrm{s}} \operatorname{sign}\left(\tau^{\mathrm{s}}\right)
$$


Tableau 1. Paramètres matériau utilisés [12].

\begin{tabular}{ccccccccc}
\hline$K\left[\mathrm{MPa} . \mathrm{s}^{\frac{1}{n}}\right]$ & $n[-]$ & $r_{\mathrm{O}}[\mathrm{MPa}]$ & $Q[\mathrm{MPa}]$ & $b[-]$ & $c[\mathrm{MPa}]$ & $d[-]$ \\
\hline \multicolumn{10}{c}{8} & 20 & & 15 & 4 & 12 & 32000 & 900 \\
\hline \multicolumn{10}{c}{$h_{0}$} & $h_{1}$ & $h_{2}$ & $h_{3}$ & $h_{4}$ & $h_{5}$ & $C_{11}[\mathrm{GPa}]$ & $C_{22}[\mathrm{GPa}]$ & $C_{44}[\mathrm{GPa}]$ \\
\hline 1 & 1 & 0,2 & 90 & 3 & 2,5 & 159,0 & 121,9 & 80,9 \\
\hline
\end{tabular}

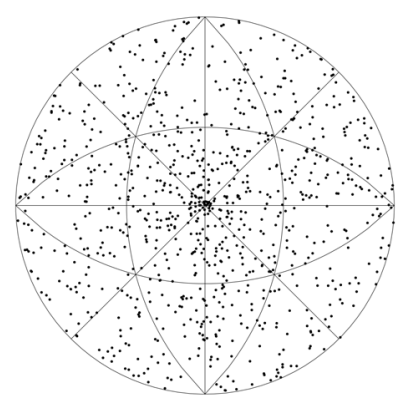

(a) Orientation 1

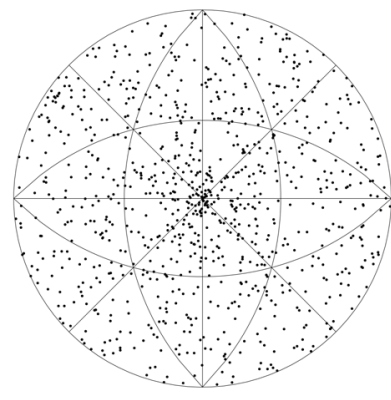

(b) Orientation 2

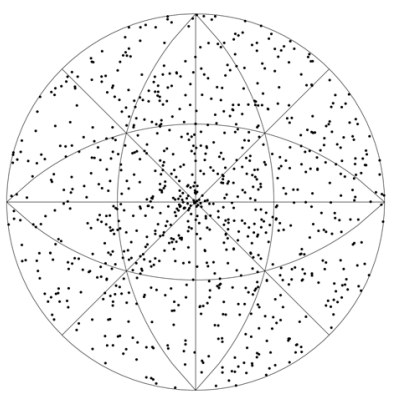

(c) Orientation 3

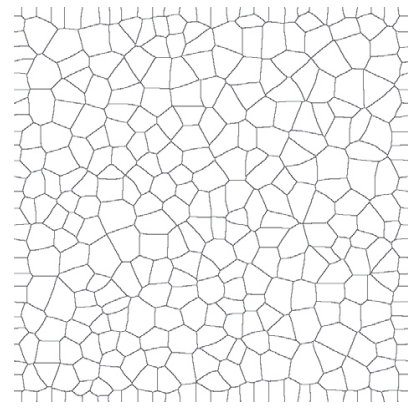

(d) Topologie 1

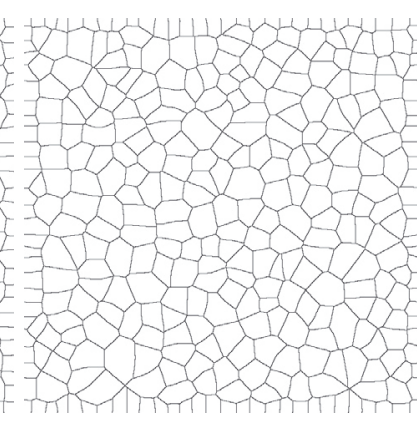

(e) Topologie 2

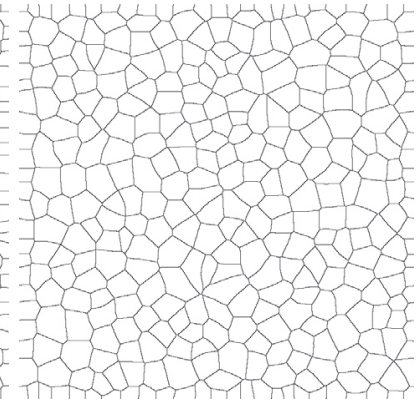

(f) Topologie 3

Fig. 2. Figures de pôles (100) pour les 3 jeux d'orientation $(\mathrm{a}-\mathrm{c})$ et les 3 topologies $(\mathrm{d}-\mathrm{f})$.

$$
\begin{gathered}
x^{\mathrm{s}}=c \alpha^{\mathrm{s}} \text { avec } \dot{\alpha}^{\mathrm{s}}=\dot{\gamma}^{\mathrm{s}}-d \dot{\nu}^{\mathrm{s}} \alpha^{\mathrm{s}} \\
r^{\mathrm{s}}=r_{0}+Q \sum_{r} h^{\mathrm{rs}}\left(1-\exp \left(-b \nu^{\mathrm{r}}\right)\right) \\
\boldsymbol{m}^{\mathrm{s}}=\left(\boldsymbol{n}^{\mathrm{s}} \otimes \boldsymbol{l}^{\mathrm{s}}+\boldsymbol{l}^{\mathrm{s}} \otimes \boldsymbol{n}^{\mathrm{s}}\right) / 2 \\
\tau^{\mathrm{s}}=\boldsymbol{m}^{\mathrm{s}}: \boldsymbol{\sigma} \\
\dot{\boldsymbol{\varepsilon}}^{\mathrm{pl}}=\sum_{s} \dot{\gamma}^{\mathrm{s}} \boldsymbol{m}^{\mathrm{s}}
\end{gathered}
$$

\section{Modèle numérique et chargements}

Les agrégats polycristallins considérés dans ce travail sont soumis à un niveau de chargement correspondant à la limite de fatigue à $10^{7}$ cycles. Les données expérimentales issues des travaux de [13] sont utilisées afin d'identifier les deux coefficients du critère de Crossland. Le seuil du critère de Crossland est défini par $\tau_{\text {oct, a }}=-0,1615 \sigma_{\text {hyd, } \max }+29,5$, avec $\tau_{\text {oct, a }}$ l'amplitude de la cission octahédrique et $\sigma_{\text {hyd, max }}$ la contrainte hydrostatique maximale. Ce critère est alors utilisé (de manière arbitraire) pour définir les niveaux de chargement à appliquer en traction, torsion et traction torsion combinées en phase. Le rapport de charge $R_{\sigma}=\frac{\sigma_{\mathrm{a}, \min }}{\sigma_{\mathrm{a}, \max }}$ est de -1 et dans le cas de la traction torsion combinées, le rapport de biaxialité $\frac{\sigma_{a}}{\tau_{2}}=1$. Les limites de fatigue déduites valent $56 \mathrm{MPa}$ en traction alternée, $36 \mathrm{MPa}$ en torsion et $30 \mathrm{MPa}$ en traction torsion combinées.

Pour tous les cas de chargements étudiés, trois topologies de microstructures contenant 300 grains équiaxes et associées chacune à trois jeux d'orientations aléatoires des grains ont été utilisées. Pour les neuf configurations obtenues, dix cycles de chargement ont été appliqués. Les microstructures ainsi que les maillages sont périodiques suivant les 2 axes. Les différentes topologies ainsi que les différents jeux d'orientations sont présentés sur la figure 2. Les éléments finis utilisés sont des triangles à 3 nœuds en déformations planes généralisées. Le nombre moyen d'éléments par grains est environ 400.

Les conditions aux limites sont des déplacements périodiques avec déformation macroscopique imposée. 


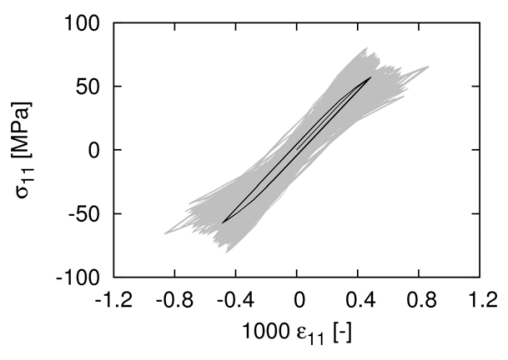

(a) Traction

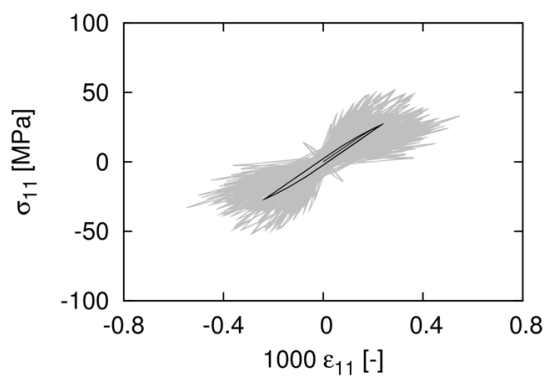

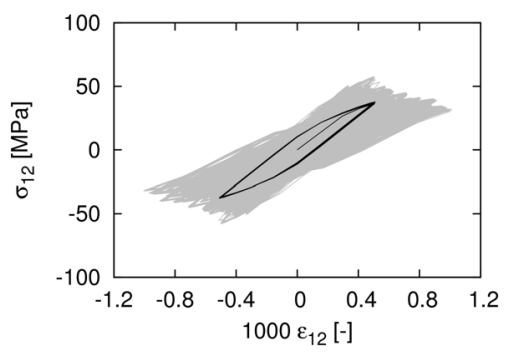

(b) Torsion

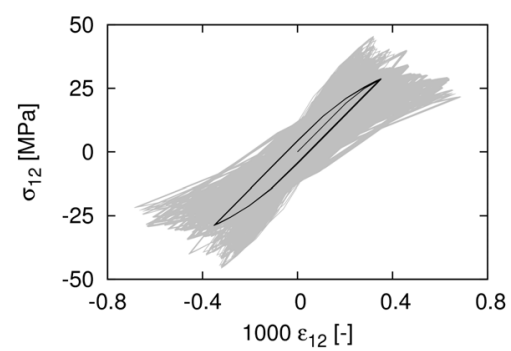

(c) Traction/Torsion

Fig. 3. Comportement cyclique à l'échelle macroscopique (en noir) et mésoscopique (en gris)

Le code éléments-finis utilisé est ZeBuLoN, développé par le Centre des Matériaux de l'École des Mines ParisTech, Northwest Numerics et l'ONERA. Le solveur implicite est employé.

\section{Résultats et analyses}

Les résultats sont présentés à l'échelle macroscopique et mésoscopique :

1. Échelle macroscopique : les quantités mécaniques sont calculées à partir du tenseur des contraintes, associé au chargement imposé;

2. Échelle mésoscopique : les quantités mécaniques sont calculées à partir de la moyenne volumique dans un grain de chaque composante du tenseur des contraintes, en excluant les éléments finis du grain ayant au moins un nœud en commun avec les grains voisins. Ceci évite de prendre en compte les concentrations de contraintes aux joints de grain dues au maillage (artefact).

La figure 3 présente les courbes contrainte/déformation aux deux échelles pour les différents cas de chargement. On constate que les cycles restent globalement centrés à l'échelle mésocopique. Le domaine des tracés en gris représente l'étendue des valeurs prises à l'échelle des grains dans le VER.

Deux critères d'endurance multiaxiaux habituellement employés dans le cadre du dimensionnement en fatigue sont appliqués aux deux échelles macroscopique et mésoscopique. Le critère de Crossland fait intervenir l'amplitude de la cission octaédrale et la contrainte hydrostatique maximale (sur la période du cycle de charge) alors que le critère de Dang Van utilise les valeurs instantanées de l'amplitude de la cission et de la contrainte hydrostatique. La figure 4 permet d'apprécier, pour le critère de Crossland, les comportements aux échelles macroscopique et mésoscopique pour les différents cas de chargements étudiés et pour les neuf microstructures générées numériquement. On constate une grande dispersion des valeurs obtenues à l'échelle mésoscopique. Le point représentatif de l'échelle macroscopique se situe à l'intérieur du nuage des points relatifs à chacun des grains. Les graphes des densités semblent indiquer que le point relevant de l'application à l'échelle macroscopique du critère se positionne non loin de la zone de plus grande densité à l'échelle mésoscopique. On parlera alors plutôt d'une bonne représentation « en moyenne » de la réponse à l'échelle mésoscopique.

La figure 5 est, elle, associée au critère de Dang Van. On observe à nouveau une grande dispersion des quantités mécaniques locales. La cission résolue maximale sur tous les systèmes de glissements simulés à l'échelle mésoscopique semble néanmoins très proche de la valeur maximale de la cission à l'échelle macroscopique. Il n'en va pas de même pour la contrainte hydrostatique macroscopique maximale qui se situe bien en deçà des quantités mésoscopiques obtenues. Les densités permettent également de mettre en évidence que la forme du trajet de chargement macroscopique dans l'espace du critère est en accord avec les tendances moyennes à l'échelle mésoscopique. 


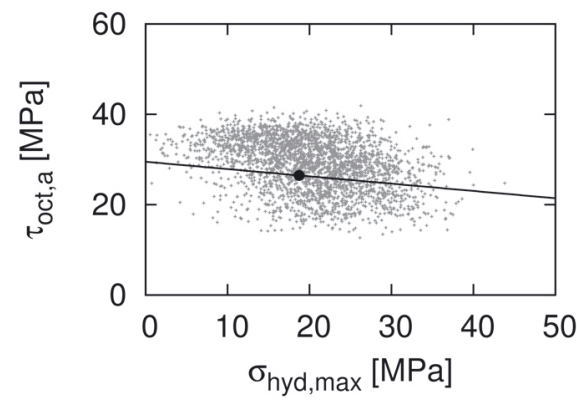

(a) Traction

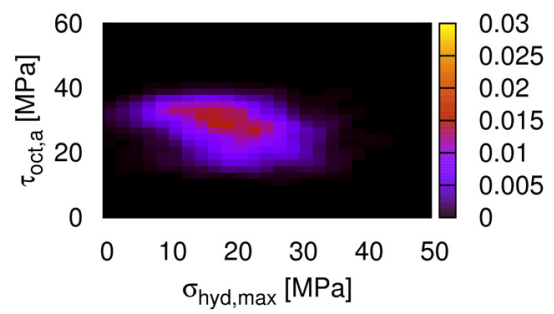

(d) Traction

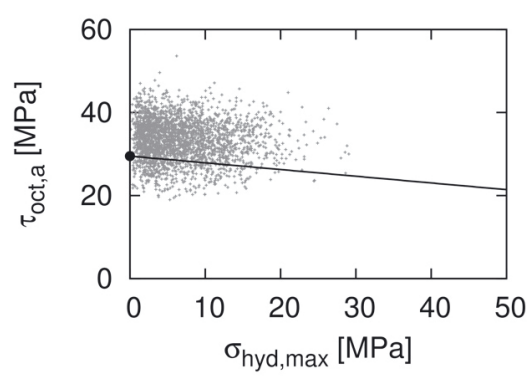

(b) Torsion

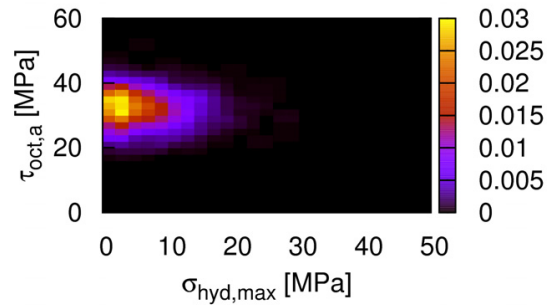

(e) Torsion

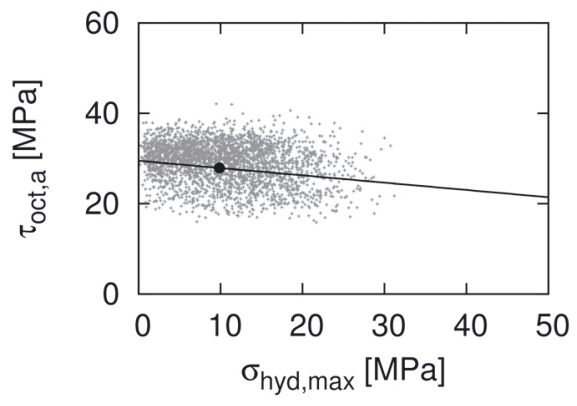

(c) Traction/Torsion

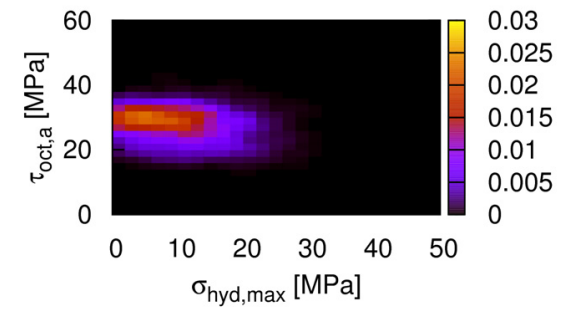

(f) Traction/Torsion

Fig. 4. Critère de Crossland pour les différents cas de chargement. (a-c) En noir, l'échelle macroscopique, en gris l'échelle mésoscopique pour les différents agrégats (300 grains $\times 9$ microstructures). (d-f) Densité à l'échelle mésoscopique.

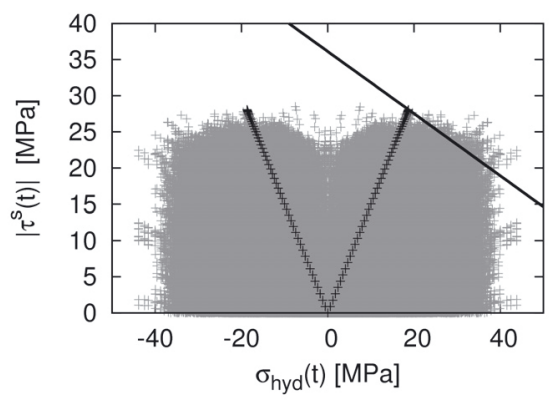

(a) Traction

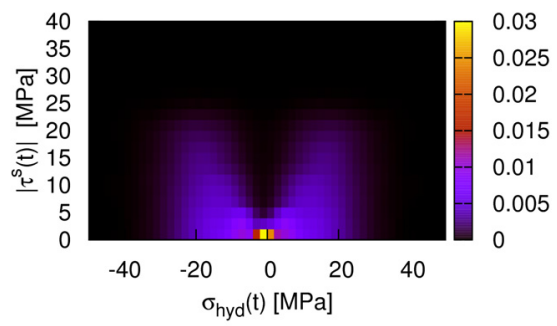

(d) Traction

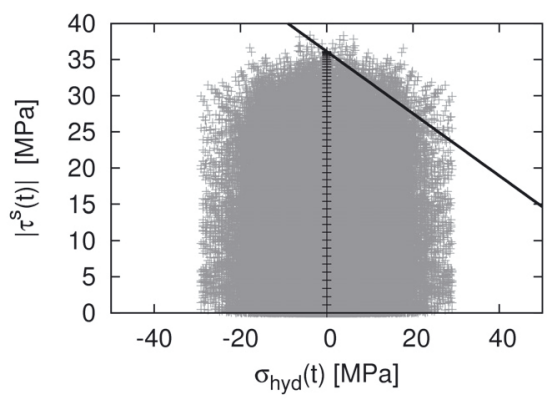

(b) Torsion

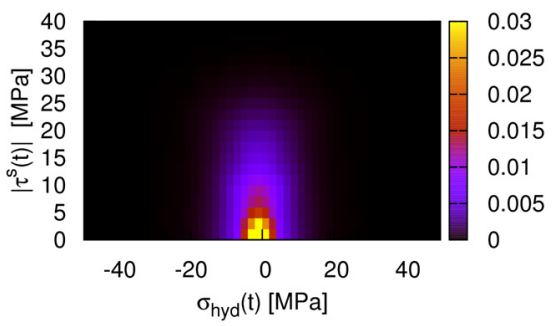

(e) Torsion

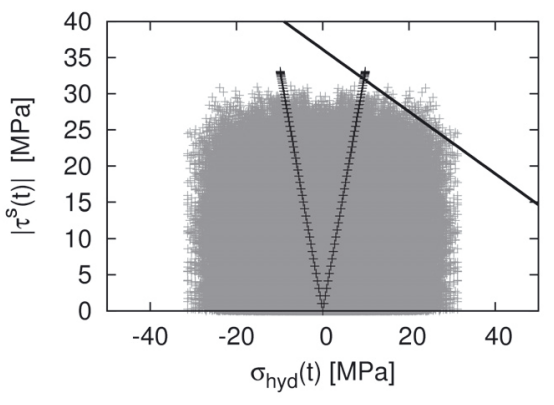

(c) Traction/Torsion

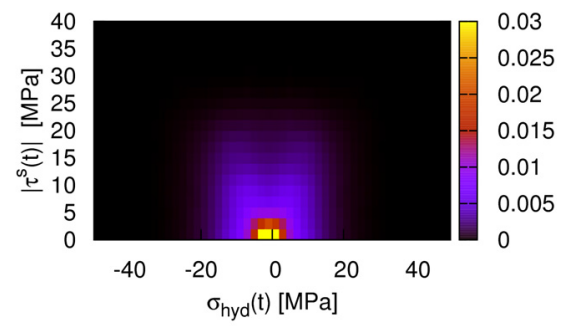

(f) Traction/Torsion

Fig. 5. Critère de Dang Van. En noir, l'échelle macroscopique, en gris l'échelle mésoscopique pour les différents agrégats (12 systèmes de glissement $\times 300$ grains $\times 9$ microstructures $)$. $(\mathrm{d}-\mathrm{f})$ densité à l'échelle mésoscopique. 
L'application de ces deux critères aux deux échelles permet de mettre en évidence une sous-estimation plus ou moins importante de la prédiction macroscopique par rapport aux points les plus critiques relatifs à l'échelle mésoscopique. Le critère de Crossland présente les écarts les plus importants. Le critère de Dang Van est plus proche de la réponse à l'échelle mésoscopique au moins en ce qui concerne la cission résolue maximale atteinte, mais pas la contrainte hydrostatique maximale.

Pour les deux critères, il semble donc que le seuil d'endurance défini à l'aide de quantités macroscopiques sous-estime les valeurs maximales des quantités locales obtenues via la simulation. Ces écarts proviennent du comportement élastique anisotrope des grains mais également des contraintes résiduelles issues de la distribution hétérogène des déformations plastiques dans l'agrégat polycristallin.

\section{Conclusion}

Une analyse critique de deux critères d'endurance multiaxiaux, critère de Crossland et critère de Dang Van a été proposée en s'appuyant sur des résultats de simulation numérique d'agrégats polycristallins. L'application des critères aux échelles macroscopique et mésoscopique met clairement en évidence le rôle joué par la microstructure (morphologie, orientation, comportement des grains) sur les écarts observés entre les deux échelles. On constate en particulier, pour différents chargements macroscopiques imposés (traction, torsion, traction torsion combinées), que les quantités macroscopiques sousestiment les valeurs maximales obtenues dans les grains. La contrainte hydrostatique mésoscopique maximale est, en particulier, bien plus grande que son équivalent macroscopique.

\section{Références}

[1] L. Meric, G. Cailletaud, Single crystal modeling for structural calculations: Part 2, finite element implementation, J. Eng. Mater. Technol. 113 (1991) 171-182
[2] F. Bridier, D.L. McDowell, P. Villechaise, J. Mendez, Crystal plasticity modeling of slip activity in ti-6al-4v under high cycle fatigue loading, Int. J. Plasticity 25 (2009) 1066-1082

[3] F. Morel, N. Huyen, Plasticity and damage heterogeneity in fatigue, Theor. Appl. Fract. Mech. 49 (2008) 98-127

[4] M. Poncelet, C. Doudard, S. Calloch, B. Weber, F. Hild, Probabilistic multiscale models and measurements of selfheating under multiaxial high cycle fatigue, J. Mech. Phys. Solids 58 (2010) 578-593

[5] S. Weyer, A. Fröhlich, H. Riesch-Oppermann, L. Cizelj, M. Kovac, Automatic finite element meshing of planar Voronoi tessellations, Eng. Fract. Mech. 69 (2002) 945958

[6] K.S. Zhang, M.S. Wu, R. Feng, Simulation of microplasticity-induced deformation in uniaxially strained ceramics by 3-d Voronoi polycrystal modeling, Int. J. Plast. 21 (2005) 801-834

[7] T. Luther, C. Könke, Polycrystal models for the analysis of intergranular crack growth in metallic materials, Eng. Fract. Mech. 76 (2009) 2332-2343

[8] M. Groeber, S. Ghosh, M.D. Uchic, D.M. Dimiduk, A framework for automated analysis and simulation of 3d polycrystalline microstructures. Part 2: Synthetic structure generation, Acta Mater. 56 (2008) 1274-1287

[9] L. St-Pierre, E. Héripré, M. Dexet, J. Crépin, G. Bertolino, N. Bilger, 3d simulations of microstructure and comparison with experimental microstructure coming from o.i.m analysis, Int. J. Plast. 24 (2008) 15161532

[10] Y. Bhandari, S. Sarkar, M. Groeber, M.D. Uchic, D.M. Dimiduk, S. Ghosh, 3d polycrystalline microstructure reconstruction from fib generated serial sections for fe analysis, Comput. Mater. Sci. 41 (2007) 222-235

[11] F. Barbe, S. Forest, G. Cailletaud, Intergranular and intragranular behavior of polycrystalline aggregates. Part 2: Results, Int. J. Plast. 17 (2001) 537-563

[12] C. Gérard, F. N'Guyen, N. Osipov, G. Cailletaud, M. Bornert, D. Caldemaison, Comparison of experimental results and finite element simulation of strain localization scheme under cyclic loading, Comput. Mater. Sci. 46 (2009) 755-760 Proceedings of the 18th International Workshop on Computational Mechanics of Materials, IWCMM-18.

[13] P. Lukás, L. Kunz, Effect of mean stress on cyclic stressstrain response and high cycle fatigue life, Int. J. Fatigue 11 (1989) 55-58 